\title{
LETTER
}

\section{The curse of the Pharaoh revisited: evolutionary bi-stability in environmentally transmitted pathogens}

Benjamin Roche, ${ }^{1,2 *}$ John M. Drake $^{3}$ and Pejman Rohani ${ }^{1,4,5}$

\begin{abstract}
It is increasingly evident that for a number of high-profile pathogens, transmission involves both direct and environmental pathways. Much of the distinguished evolutionary theory has, however, focused on each of transmission component separately. Herein, we use the framework of adaptive dynamics to study the evolutionary consequences of mixed transmission. We find that environmental transmission can select for increased virulence when direct transmission is low. Increasing the efficiency of direct transmission gives rise to an evolutionary bi-stability, with coexistence of different levels of virulence. We conclude that the overlooked contribution of environmental transmission may explain the curious appearance of high virulence in pathogens that are typically only moderately pathogenic, as observed for avian influenza viruses and cholera.
\end{abstract}

\section{Keywords}

Adaptative dynamics, curse of the pharaoh, environmental transmission, evolutionary bi-stability.

\section{INTRODUCTION}

Classical evolutionary theory holds that selection will cause virulence of a directly transmitted pathogen to stabilize at an intermediate optimum because of the trade-off between pathogen replication and illness induced host behaviours that reduce transmission (Anderson \& May 1982b; Dieckmann et al. 2002; Alizon et al. 2009). Instances of rapid evolution in introduced pathogens, for example the Myxoma virus in Australian rabbits (Fenner \& Ratcliffe 1965), have been interpreted to provide broad support for this theory. By contrast, indirectly transmitted pathogens in which the lifespan of the infectious particles in the environment exceeds the infectious period of the host are predicted not to be constrained by this trade-off, and therefore enabled to maintain a greater virulence. This phenomenon has been called the 'curse of the Pharaoh' (Bonhoeffer et al. 1996) in reference to the mysterious death of Lord Carnavon upon the opening of the sarcophagus of Tutankhamen (Corelli 1923). Consistent with this prediction, comparative studies suggest that pathogens whose life cycle includes a free-living stage typically inflict higher virulence on their hosts than directly transmitted pathogens (Walther \& Ewald 2004). To explain this pattern, it has been suggested that environmentally mediated transmission reduces the dependence of the pathogen on host availability and that a predominantly environmentally transmitted pathogen can 'sit' within the environment and 'wait' for new susceptibles to infect (Ewald 1987, 1991, 1993, 1994; Walther \& Ewald 2004), thereby substantially reducing the costs associated with high virulence. The predictions of this verbal argument have been called into question by prior theoretical studies. For instance, Bonhoeffer et al. (1996) showed that virulence should be unaffected by propagule longevity in the environment [note that it has been shown how the relaxation of certain assumptions in the Bonhoeffer et al. (1996) study can lead to increased virulence (Gandon 1998; Caraco \& Wang 2008)].

How do predictions of theory based on direct transmission alone or derived from sit-and-wait strategies apply to infectious diseases with two distinct transmission modes? This distinction is important because in such systems, transmission dynamics involve two time scales: (1) one fast, reminiscent of direct transmission, resulting from the heterogeneous clumped concentration of infectious particles that are deposited in the environment and encountered by susceptible hosts in groups, and (2) a slower time scale, resulting from the accumulated contamination of the host environment as centres of particle concentration break down and are redistributed, resulting in chronic exposure. Such 'mixed transmission' pathogens include Vibrio cholerae (King et al. 2008; de Magny et al. 2008), Methicillin-resistant Staphylococcus aureus [MRSA, Eveillard et al. (2004)], smallpox (Walther \& Ewald 2004), Group A streptococcus (Beres et al. 2002), and avian influenza viruses (Webster et al. 1992; Roche et al. 2009; Rohani et al. 2009). In these cases, transmission should be represented by two independent processes whose rates scale with the duration of infection and the persistence of infectious particles in the environment, respectively (Rohani et al. 2009).

Herein, we study a model for the evolution of such a pathogen. We show that environmental transmission can select for higher virulence, but only if the relative contribution of direct transmission (the fast component) is small. In contrast, if the mix between fast and slow infection is sufficiently balanced, an evolutionary bi-stability emerges. These results show that considering dual transmission routes

\footnotetext{
${ }^{1}$ Department of Ecology and Evolutionary Biology, University of Michigan,

Ann Arbor, MI 48109, USA

${ }^{2}$ UMI IRDIUPMC 209 - UMMISCO, 93143, Bondy, France

${ }^{3}$ Odum School of Ecology, University of Georgia, Athens, GA 30602, USA

${ }^{4}$ Center for the Study of Complex Systems, University of Michigan, Ann Arbor,

MI 48109 USA
}

\author{
${ }^{5}$ Fogarty International Center, National Institutes of Health, Bethesda, \\ MD 20892, USA \\ *Correspondence: E-mail: benjamin.roche@ird.fr
}


leads to a qualitatively different evolutionary outcome than each route considered in isolation. Our findings might explain the frequent coexistence of high and low virulence strains of such pathogens as avian influenza viruses (Webster et al. 1992) and cholera (Cvjetanovic \& Barua 1972).

\section{OUR MODEL}

Our model is motivated by previous work on the transmission dynamics of cholera (Codeço 2001; Pascual et al. 2002) and avian influenza viruses (Webster et al. 1992; Roche et al. 2009; Rohani et al. 2009; Breban et al. 2010; Roche \& Rohani 2010). The model is an extension of the SIR framework, in which individuals are classified according to infectious status as Susceptible, Infectious or Recovered (Dietz 1976; Anderson \& May 1991; Keeling \& Rohani 2008). We add to this model a process of slow, environmentally mediated transmission and the competition of $n$ pathogen strains (denoted by subscript $\imath$ ), leading to the following system of equations:

$\frac{d S}{d t}=v-\sum_{i=1}^{n}\left(\lambda_{i} S\right)-\mu S$

$\frac{d I_{i}}{d t}=\lambda_{i} S-\left(\mu+\gamma_{i}+\alpha_{i}\right) I_{i}$

$\frac{d V_{i}}{d t}=\omega_{i} I_{i}-\eta_{i} V_{i}$

where $\lambda_{i}$ is the strain-specific force of infection and is given by:

$\lambda_{i}=\sigma \beta_{i} I_{i}+\rho \delta_{i} \frac{V_{i}}{V_{i}+\kappa_{i}}$.

Here, $v$ represents the population birth rate, $\mu$ is the per capita death rate, and $\gamma_{i}$ and $\alpha_{i}$ are the strain-specific host recovery rate and pathogen virulence (disease-induced mortality), respectively. Transmission of the pathogen to susceptibles may occur directly with density-dependent force of infection $\left(\sigma \beta_{i} I_{i}\right)$, where $\sigma$ represents the host contact rate and $\beta_{i}$ denotes infectiousness, or indirectly via chronic exposure to the contaminated environment, with force of infection $\left(\rho \delta_{i} \frac{V_{i}}{V_{i}+\kappa_{i}}\right)$, where $\rho$ is the uptake rate of the environmental reservoir and $\kappa_{i}$ denotes the strain-specific dose that yields a $50 \%$

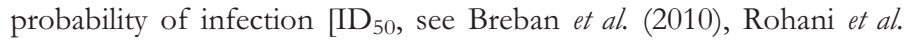
(2009)]. The term $\delta_{i}$ is the fraction of infections due to strain $i$, here interpreted as a probability (Breban et al. 2010; Roche \& Rohani 2010):

$\delta_{i}=\frac{V_{i} / \kappa_{i}}{\sum V_{i} / \kappa_{i}}$.

Finally, infectious individuals $\left(I_{i}\right)$ shed particles into the environmental reservoir $\left(V_{i}\right)$ at rate $\omega_{i}$ which decay at rate $\eta_{i}$. Considered on a particle by particle basis, this assumption implies that the average duration of infectiousness in free-living particles is $1 / \eta_{i}$. For concreteness, we assume perfect cross-immunity between strains (Breban et al. 2010). A generalized version of this model with mutations to allow strain evolution is presented in the Supporting Information Appendix S1.

Compartmental models such as this typically yield a threshold, referred to as $R_{0}$, that determines the stability of an interior equilibrium corresponding to endemicity of the pathogen. It has previously been shown for a single strain with this transmission structure that $R_{0}$ is the sum of the $R_{0}$ s for the direct $\left(R_{0}^{d i r}\right)$ and indirect $\left(R_{0}^{e n v}\right)$ transmission components:

$R_{0}^{[i]}=R_{0}^{\operatorname{dir}[i]}+R_{0}^{e n v[i]}=\frac{N \sigma \beta_{i}}{\gamma_{i}+\mu+\alpha_{i}}+\frac{N \rho \omega_{i}}{\eta_{i} \kappa_{i}\left(\gamma_{i}+\mu+\alpha_{i}\right)}$

respectively (Rohani et al. 2009). In this equation, $N$ denotes the host population size at the disease-free equilibrium and is given by $v / \mu$.

Given such a model for transmission, the evolutionary strategy of strain $i$ may be defined as the 4-tuple $\left(\beta_{i}, \alpha_{i}, \omega_{i}, \eta_{i}\right)$, with other parameters assumed not to be subject to selection. Then, an evolutionarily stable strategy (ESS) is defined as a strategy that must be able to resist invasion by mutants (Maynard-Smith 1982; Nowak 2006). In mathematical terms, an ESS has to satisfy the following conditions (Dieckmann et al. 2002):

$\Re_{0}\left(z, z^{*}\right)=\frac{\omega^{*} \rho \hat{S}}{\eta^{*}\left(\mu+\gamma^{*}+\alpha^{*}\right)(\hat{V}+1)}+\frac{\beta^{*} \sigma \hat{S}}{\mu+\gamma^{*}+\alpha^{*}}$,

where $\Re_{0}\left(z z^{*}\right)$ is the fitness of a mutant strategy (depicted by $z^{*}$ ) invading a population with the resident allele ( $z$ ) (Breban et al. 2010). $\hat{S}$ and $\hat{V}$ are, respectively, the endemic equilibria of susceptibles and the environmental concentration of the resident strain. In contrast to coexistence studies that consider only the conditions required for invasion, i.e. when $\Re_{0}\left(z, z^{*}\right)>1$ (Breban et al. 2010), we aim here to determine strategies which resist invasion, i.e. when $d \AA_{0}\left(z, z^{*}\right) / d z^{*}=$ 0 and $d^{2} \Re_{0}\left(z, z^{*}\right) / d z^{*}<0$ at $z^{*}=q$. Hence, ESSs represent strategies favoured by natural selection (Parker \& Smith 1990; Dieckmann et al. 2002).

\section{Trade-offs}

To address the trade-off between pathogen replication and transmissibility, we introduce constraints to the set of possible evolutionary strategies. Specifically, similar to previous theoretical studies, we assume that shedding rate $\left(\omega_{i}\right)$, infectiousness $\left(\beta_{i}\right)$, and virulence $\left(\alpha_{i}\right)$ all increase with an unobserved quantity $\%$. The trade-off functions we incorporate are inspired by the empirical results derived from a phage-bacterium system, which showed that greater phage production is associated with reduced environmental persistence (De Paepe \& Taddei 2006), i.e. greater host exploitation comes at a cost realized in terms of environmental persistence $\left(1 / \eta_{i}\right)$. For concreteness, these trade-offs are characterized by a system of non-decreasing shifted exponentials,

$\beta(z)=a\left(1-e^{-b z}\right)$

$\omega(z)=c+d e^{f e^{-g z}}$

$\eta(z)=h+i\left(1-e^{-j z}\right)$

where $a, b, c, d, f, g, h, i$ and $j$ are constants (Fig. 1). Note that the shedding rate, $\omega$, is a Gompertz function, which causes $R_{0}^{e n v}$ to be smooth, but non-monotonic in $\%$, inducing two different strategies that exploit environmental transmission (Fig. 1, bottom panel). To represent evolutionary constraints due to virulence, we set $z=\alpha$, reflecting that shedding and infectiousness increase and durability $(1 / \eta)$ decreases with within-host replication. From this parameterization, we obtain two results. First, a low virulence, steady-and-slow strategy with low infectiousness and low shedding occurs at the extreme of avirulence (note the local mode in $R_{0}$ as $\alpha \rightarrow 0$ ). The second strategy is more aggressive, with high infectiousness and 


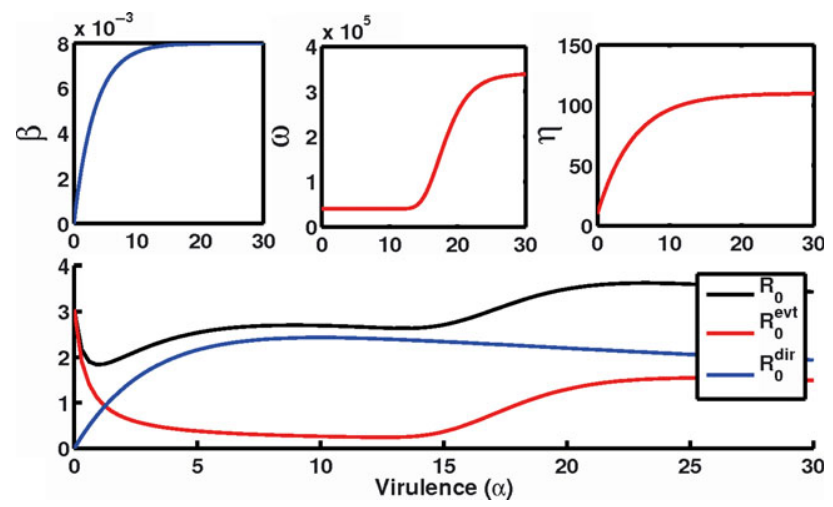

Figure 1 Trade-off shapes and $R_{0}$ according to $\alpha_{i}$ (The population is entirely susceptible and only one strain is considered) in the case of virulent strategies $\left(\alpha_{i}=\right.$ zi). Trade-off parameters values: $a=0.008, b=0.3, c=4.10^{4}, d=3.10^{5}, f=$ $-1000, g=0.4, \quad h=10, \quad i=100, j=0.2$. Epidemiological and ecological parameters: $\rho=4.10^{-7} \mathrm{~L}$ year ${ }^{-1}, \sigma=2$ year $^{-1}, \mu=0.5$ year $^{-1}, v=5000$ year $^{-1}$, $N=10000, \kappa=10 E^{E} D_{50}, \gamma=1 / 7$ days.

shedding of a highly virulent pathogen transmitted primarily through the environmental pathway despite short persistence in the environment (mode in $R_{0}$ around $\alpha=25$ in Fig. 1, bottom panel). Next, foreshadowing an evolutionary bi-stability that can occur in this system, we note that previous analyses of coexistence in mixed transmission systems have found that a single dominant strategy would emerge, driving all others to extinction (Breban et al. 2010; Roche \& Rohani 2010). The inclusion of pathogen virulence is a key difference between our model and these previous studies. Anticipating that to understand the ecological effects of disease-induced mortality on this evolutionary bi-stability, we will want to compare virulent and avirulent strains while retaining evolutionary constraints. Accordingly, we set $\alpha=0$ (null virulence) and interpret $z$ as a constraint on $\beta, \omega$ and $\eta$ that does not increase mortality, effectively decoupling virulence and the trade-off itself (see Appendix S2).

\section{Analysis}

The evolutionary dynamics implied by this model is summarized in three sets of results. First, analysis of the mixed transmission system reveals $0,1,2$ or 3 ESSs are possible. Second, inspection of Pairwise Invasion Plots (PIPs) shows where evolutionary bi-stability is expected to occur. Finally, a focus on the effect of virulence on evolutionary bi-stability demonstrates the conditions under which two strains differing in virulence may stably coexist. In particular, we show that removing the selection pressure induced by pathogen virulence excludes the possibility of a stable polymorphism.

\section{ESS for mixed transmission strains}

Evolutionarily stable strategys of this system are most compactly represented as a function of the host contact rate, $\sigma$ and the environmental uptake rate, $\rho$. Under these conditions, four scenarios emerge (Fig. 2). The details of each scenario are visualized by its PIP which catalogues the invasibility of different resident and mutant strategies (Fig. 3).

The first scenario (labelled region A in Fig. 2) occurs when contact rate is high and uptake rate is low, leading to the classical result, a

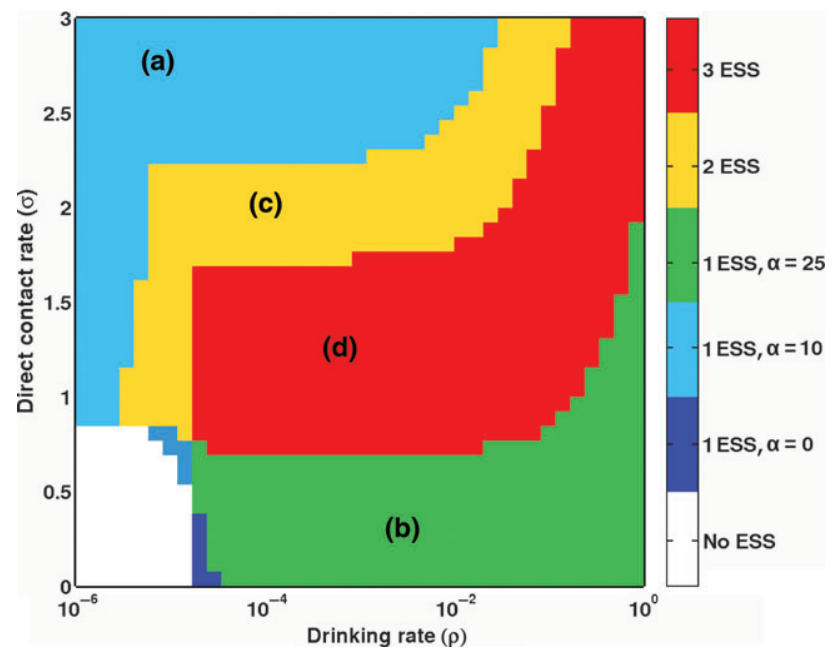

Figure 2 Number of Evolutionary Stable Strategies as a function of host contact rate and environmental uptake rate obtained by maximizing the fitness function (denoted in eqn 6) where the resident strain was at endemic equilibrium. Other parameters are the same as before: $a=0.008, b=0.3, c=4.10^{4}, d=3.10^{5}, f=$ $-1000, g=0.4, \quad b=10, \quad i=100, j=0.2$. Epidemiological and ecological parameters: $\mu=0.5$ year $^{-1}, v=5000$ year $^{-1}, N=10000, \kappa=10$ EID $_{50}, \gamma=$ $1 / 7$ days.

single globally stable ESS at an intermediate level of virulence (Anderson \& May 1982b; Alizon et al. 2009). This represents the best host exploitation strategy since the cost of virulence is compensated by gains in direct transmission. Any strain introduced to this system, regardless of its strategy, evolves to this level of virulence (Fig. 3a).

In contrast, when the environmental uptake rate is high and the host contact rate is low, two locally stable ESSs occur (region B in Fig. 2). As shown in Fig. 3(b), here, two different levels of virulence can emerge: an avirulent strategy, with low viral shedding rate and durability in the environment, and a virulent strategy, where rates of shedding and degradation are high. This bi-stability arises at the evolutionary repeller (Fig. 3b, red circle) where the resident strategy can be invaded by less and more virulent mutant strategy (Dieckmann et al. 2002). Hence, when a strain is introduced at this repeller, its strategy may evolve to either of the local ESSs (Fig. 3b, blue circles).

As host contact rates are increased (region $\mathrm{C}$ in Fig. 2), two other locally stable ESSs emerge (Fig. 3c, blue circles). Here, the high virulence strategy from scenario B is no longer an ESS and is replaced by the intermediate virulence strategy from scenario A because of the faster epidemic growth associated with direct transmission (Rohani et al. 2009).

Finally, for intermediate contact and uptake rates, we observe three distinct locally stable ESSs (region D in Fig. 2). The stable points are a combination of the scenarios dominated by direct and environmental transmission, respectively (Fig. 3d). As with scenario B, we expect an evolutionary bi-stability. By contrast, however, the locally stable ESSs surrounding this bistable solution are the virulent strategy from environmental contamination and the intermediate virulence strategy from direct transmission (Fig. 3d).

\section{Evolutionary dynamics and evolutionary bi-stability}

Having identified the conditions under which evolutionary bi-stability can occur, we now turn to the evolutionary dynamics of virulence in 


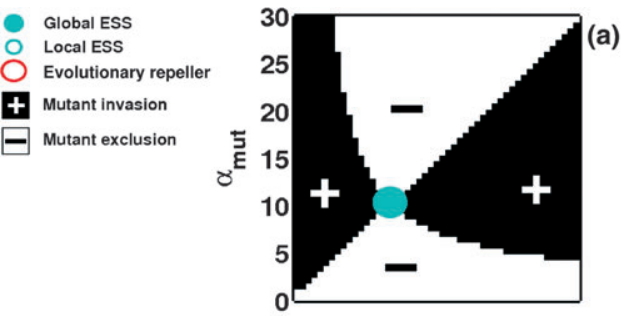

(a)
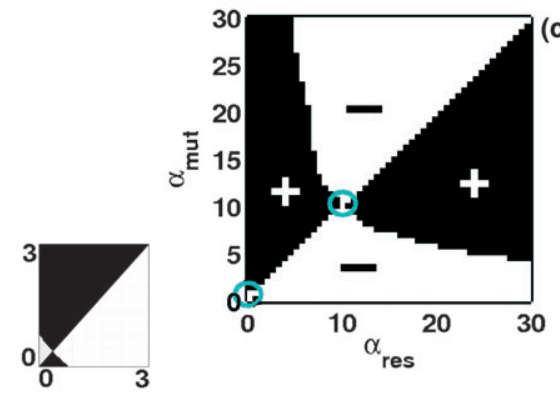

(c)

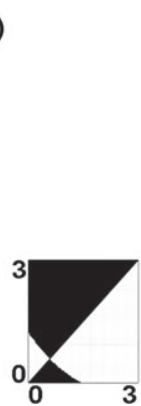

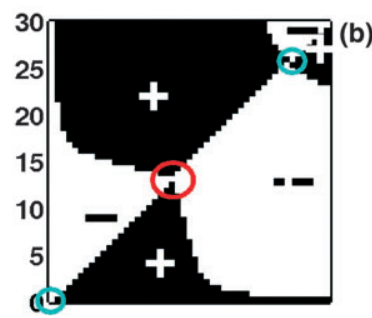

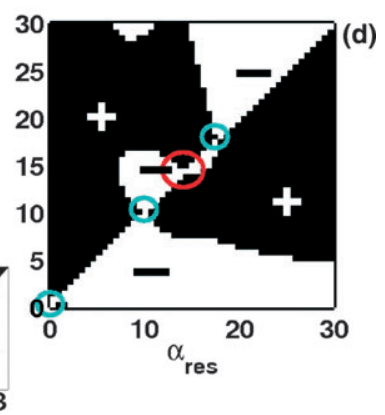

Figure 3 Pairwise Invasion Plots (PIP) for the scenarios depicted in Fig. 2. Points identify the globally stable (filled blue), locally stable (blue) and unstable (red) evolutionary strategies in four scenarios: (a) Only direct-transmission $(\sigma=3, \rho=0)$, (b) Only environmental transmission $\left(\sigma=0, \rho=2 \cdot 10^{-2}\right)$. (c) Direct transmission is high and environmental transmissions is moderate $\left(\sigma=2, \rho=2 \cdot 10^{-2}\right)$. (d) Both direct and environmental transmission are moderate $\left(\sigma=1.6, \rho=2.10^{-2}\right)$. Other parameters are as before (Trade-off parameters: $a=0.008, b=0.3, c=$ $4.10^{4}, d=3.10^{5}, f=-1000, g=0.4, b=10, i=100, j=0.2$; Ecological parameters: $\mu=0.5$ year $^{-1}, v=5000$ year $^{-1}, N=$ $10000, \kappa=10 E I D_{50}, \gamma=1 / 7$ days). scenarios B and D. Our primary aim in this section is to understand the feedback between transient epidemiological dynamics and evolution. Numerical experiments using the evolutionary model (Appendix S1) starting with a uniform distribution of virulence across the interval $[0,30]$, no mutation, and only environmental transmission resulted in competitive exclusion of all phenotypes except the avirulent strain (Fig. 4a), consistent with previous studies (Breban et al. 2010; Roche \& Rohani 2010). Importantly, the addition of continuous mutation changes this picture. Now, two basins of attraction emerge: trajectories converge on avirulence when the introduced strain has low virulence $\left(\alpha_{i}(t)<15\right.$, Fig. 4b), whereas, as anticipated by the pairwise invasion analysis (Fig. 3b), the majority of trajectories converge to a stationary distribution of positive virulence if the founding strain is even weakly virulent $\left(\alpha_{i}(t)>15\right.$, Fig. $\left.4 \mathrm{c}\right)$. We reiterate that this phenomenon is fully created by the addition of mutation, since the model without mutation evolved to avirulence when all possible strategies were introduced (sensitivity of our results with different mutation process is discussed in Appendix S4).

We now examine this phenomenon when the model is extended to include direct transmission. Again, we first assume no mutation, when all strains are initially introduced. In this case, in contrast to the scenario with environmental transmission alone (Fig. 4d), the system evolves to stable coexistence of two moderately virulent strains (Fig. 4d). With mutation, an evolutionary bifurcation occurs (Fig. 4e) yielding two ESSs. Note that our pairwise invasibility analysis for the mixed transmission system (scenario D) predicted three ESSs (the two
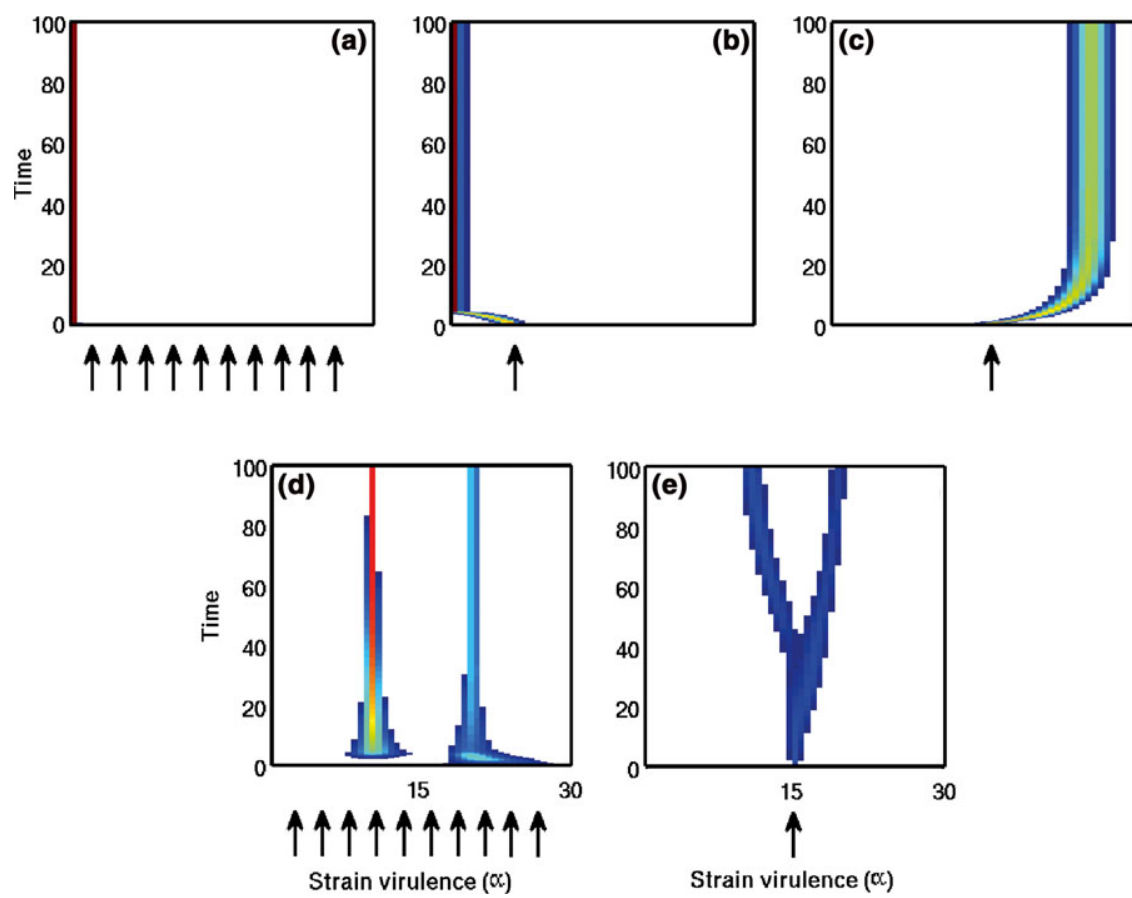

Strain virulence $(\alpha)$
Figure 4 Evolutionary dynamics by mutation between neighbouring strains. (a) Only environmental transmission is involved ( $\sigma=$ $\left.0, \rho=2 \cdot 10^{-2}\right)$, mutations do not occur $(m=0)$ and all the strains are introduced at the start of simulation. (b) Only environmental transmission is involved, mutation can occur and a strain is introduced at $\alpha=10$. (c) Similar than (b) with a strain introduced at $\alpha=20$. (d) Both transmission routes are involved ( $\sigma=1.6$, $\left.\rho=2 \cdot 10^{-2}\right)$, mutations cannot occur $(m=0)$ and all the strains are introduced at the beginning of the simulation. (e) Both transmission routes are involved, mutation can occur and a strain is introduced at the evolutionary repeller $(\alpha=15)$. Trade-off parameters: $a=0.008, b=0.3, c=4.10^{4}, d=3.10^{5}, f=-1000$, $g=0.4, h=10, i=100, j=0.2$; Ecological parameters: $\mu=$ 0.5 year $^{-1}, v=5000$ year $^{-1}, N=10000, \kappa=10 E^{2} D_{50}, \gamma=$ $1 / 7$ days, $m=0.01$. 
moderately virulent strategies together with an avirulent strategy). In simulations, however, the stable avirulent strategy is not observed because it is competitively excluded by the faster epidemic growth of the two virulent strategies. Thus, we find that either the mutation process or direct transmission can induce local stability such that a virulent ESS may persist. When both mechanisms are included, the model exhibits an evolutionary bi-stability yielding a coexistence of two strains with differential virulence levels.

\section{The influence of virulence}

Previous analyses of coexistence in mixed transmission systems showed that a single dominant strategy will drive all others to extinction (Breban et al. 2010; Roche \& Rohani 2010). This prediction is clearly at odds with the present analysis in which evolutionary bistability gives rise to a stable coexistence. The key difference, of course, is the inclusion of pathogen virulence as a heritable trait. To investigate the effects of virulence on evolutionary bi-stability, we repeated our simulation study (depicted in Fig. 4e) setting $\alpha=0$ in eqns (8)-(10). As shown in Fig. 5(a,b), removing the selection pressure induced by pathogen virulence destabilizes the lower branch. Hence, the most virulent strategy involved in environmental transmission significantly decreases host abundance and prevents competitive exclusion by the other strain.

\section{CONCLUSIONS AND DISCUSSION}

Here, we have conducted a study aimed at understanding the evolutionary dynamics of host-pathogen systems with a combination of direct and environmental transmission mechanisms. Consistent with previous theoretical studies of virulence evolution, we find that in solely directly transmitted systems selection favours intermediate virulence (Figs 2 and 3a). The introduction of environmental transmission qualitatively changes this picture such that two lifehistory strategies can stably coexist (Figs $3 \mathrm{~d}$ and 4d,e). Particularly, the presence of two transmission routes can create conditions enabling the stable persistence of two strains with different virulence levels (Fig. 4e). We suggest that this occurs because the immediate decrease in host population size following introduction mitigates the explosive increase in each strain that would otherwise drive one strategy to
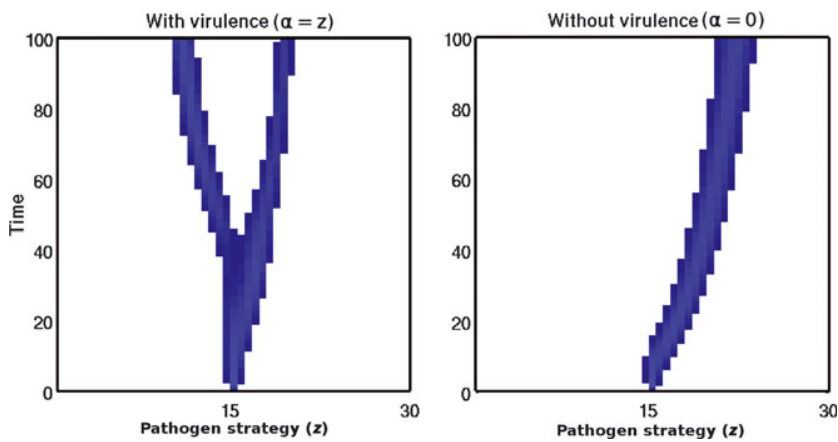

Figure 5 Evolutionary dynamics with $(\alpha=\%, \mathrm{~A})$ and without $(\alpha=0, \mathrm{~B})$ virulence. Both transmission routes are involved $\left(\sigma=1.6, \rho=2.10^{-2}\right)$ and the introduced strain is around the evolutionary repeller previously observed. Trade-off parameters: $a=0.008, b=0.3, c=4.10^{4}, d=3.10^{5}, f=-1000, g=0.4, b=10, i=$ 100, $j=0.2$. Epidemiological and ecological parameters: $\rho=4.10^{-7} \mathrm{~L}_{\text {year }}{ }^{-1}$, $\sigma=2$ year $^{-1}, \mu=0.5$ year $^{-1}, v=5000$ year $^{-1}, N=10000, \kappa=10 E^{2} D_{50}, \gamma=$ $1 / 7$ days, $m=0.01$. extinction. Hence, the evolutionary bi-stability arises because of the feedback between the epidemiological characteristics and evolutionary dynamics.

This study extends the only previous theoretical study of virulence evolution when pathogen transmission has both environmental and direct components (Day 2002). Crucially, we have relaxed the assumption in Day (2002) that propagule longevity is not a pathogen strategy. This is motivated by the work of De Paepe and Taddei (2006), describing a trade-off between environmental persistence and transmission. As we have shown, relaxing this assumption has important evolutionary consequences because it allows the emergence of two different strategies for environmentally transmitted pathogens: one prudent with long durability and another aggressive strategy with short environmental survival.

There are four key assumptions in this study that can affect our conclusions; we discuss each in turn. They are:

(1) Trade-off functions. As mentioned above, our choice of a trade-off between pathogen durability in the environment and transmission is empirically derived (De Paepe \& Taddei 2006) and, we believe, renders our conclusions applicable to numerous infectious diseases. In addition, we assume the shedding rate to be given by a Gompertz function. This results in a maximum shedding rate at a higher level of virulence than infectiousness (Fig. 1), as assumed in Day (2002). The observation that environmentally transmitted pathogens exhibit higher virulence than equivalent directly transmitted diseases (Ewald 1994) supports the premise of our assumption.

(2) Host demography. To overcome the eventual host extinction ensured by any non-zero virulence, we have assumed a constant birth rate, independent of population size. An alternative assumption would be a susceptible recruitment that is dependent on the host population size. In such a case, it would be mandatory that the per capita birth rate exceeds the per capita death rate, as is commonly observed in nature and is referred to as 'doomed surplus' (Errington 1934). It is worth pointing out that our conclusions remain qualitatively unaffected whatever is the assumption considered (Appendix S3).

(3) Strain competition and $\delta_{i}$. The outcome of environmental transmission is determined by our quantity $\delta_{i}$ (Appendix S5), which scales the relative prevalence of each strain in the reservoir $\left(V_{i}\right)$ by their infectivity $\left(\kappa_{i}\right)$. This term is important because it guarantees the 'ecological neutrality' (sensu Lipsitch et al. (2009) of our multi-strain model. Thus, if all strains are assumed equivalent, then the environmental transmission rate for the sum of the strains, $f\left(\sum_{i}^{n} V i\right)$, is identical to $\sum_{i}^{n} f\left(V_{i}\right)$.

(4) Cross-reactive immunity. We have assumed full cross-immunity between strains - that is, infection with any strain renders that host protected to other strains. While the extent and dynamics of imperfect immunity are active areas of research (Abu-Raddad \& Ferguson 2004; Koelle et al. 2006; Park et al. 2009), there is empirical support for cross-immunity among, for example, avian influenza viruses (Costa et al. 2010). We submit that partial crossimmunity would weaken the interaction conferring stability on the virulence coexistence reported herein. It follows that there is a critical level of cross-immunity at which the phenomenon will break down. This threshold can be also altered if other immunity processes are involved, such as super-infection (Nowak \& May 1994; Caraco et al. 2006) where strain competition moves from 
inter- to intra-host. What level of cross-immunity is required to enable the evolutionary bi-stability and how this relates to changes in virulence are therefore important questions for further research.

It is worth pointing out that our results can give critical insights for at least two environmentally-transmitted diseases which show, in some areas, a coexistence of two phenotypes with very different levels of virulence. Indeed, mycobacterium Vibrio cholerae, causing cholera, is naturally present in aquatic environments (Pascual et al. 2002) and a coexistence of avirulent and highly virulent variants is frequently observed (Kooper et al. 1995). In the same vein, bird populations can entertain, at the same time, 'Low Pathogenic' influenza viruses, assumed to have a negligible impact on bird fitness [Olsen et al. (2006), but see Capua \& Mutinelli (2001)], and their 'Highly Pathogenic' form (Chen et al. 2005). Both cholera and influenza can become highly virulent with the presence of a specific sequence in their genomes, i.e. a 'pathogenicity island' identified on gene CTXa for cholera (Kooper et al. 1995) and an insertion at the cleavage site in the Hemagglutinin glycoprotein for influenza (Perdue et al. 1997; Liu et al. 2005). Our study suggests that the fluctuations in environmental factors, and consequently in environmental transmission intensity, can promote the simultaneous coexistence of both phenotypes as an evolutionary stable strategy.

In this study, we have shown that the feedback between epidemiological and evolutionary dynamics, consecutive to the combination of transmission routes, can have important consequences on pathogen evolution. In wildlife species, the fluctuating nature of aquatic conditions, modifying the environmental transmission, and the unsteady contact patterns between individuals, influencing the direct contact rate, could be an important driver of the unpredictable bi-stability of pathogen virulence observed. In this context, it seems crucial to unravel this complexity by integrating the correct spillover mechanisms. Our study underlines that the consequences of environmental transmission have clearly to be considered and that it needs to become a research priority.

\section{ACKNOWLEDGEMENTS}

We thank three anonymous referees for insightful and helpful comments on this work. We also thank the Rohani and King labs at the University of Michigan for insightful comments and Guillaume Constantin de Magny for discussion on cholera. This work was supported by the Centers for Disease Control and Prevention (5U19C1000401), the James S. McDonnell Foundation and the National Science Foundation (DEB-0917853). P.R. was also supported by the RAPIDD program of the Science and Technology Directorate, Department of Homeland Security, and the Fogarty International Center, National Institutes of Health.

\section{REFERENCES}

Abu-Raddad, L.J. \& Ferguson, N.M. (2004). The impact of cross-immunity, mutation and stochastic extinction on pathogen diversity. Proc. Biol. Sci., 271, 2431-2438.

Alizon, S., Hurford, A., Mideo, N. \& Baalen, M.V. (2009). Virulence evolution and the trade-off hypothesis: history, current state of affairs and the future. J. Evol. Biol., 22, 245-259.

Anderson, R.M. \& May, R.M. (1982b). Coevolution of hosts and parasites. Parasitology, 85, 411-426.
Anderson, R.M. \& May, R.M. (1991). Infectious Diseases of Humans: Dynamics and Control. Oxford Science Publications, Oxford.

Beres, S.B., Sylva, G.L., Barbian, K.D., Lei, B., Hoff, J.S., Mammarella, N.D. et al. (2002). Genome sequence of a serotype M3 strain of group a streptococcus: phage-encoded toxins, the high-virulence phenotype, and clone emergence. Proc. Natl. Acad. Sci. U.S.A., 99, 10078-10083.

Bonhoeffer, S., Lenski, R.E. \& Ebert, D. (1996). The curse of the pharaoh: the evolution of virulence in pathogens with long living propagules. Proc. Biol. Sci., 263, 715-721.

Breban, R., Drake, J.M. \& Rohani, P. (2010). A general multi-strain model with environmental transmission: invasion conditions for the disease-free and endemic states. JTB, 264, 729-736.

Capua, I. \& Mutinelli, F. (2001). Low pathogenicity (LPAI) and highly pathogenic (HPAI) avian influenza in turkeys and chicken. In: A Colour Atlas and Text on Avian Influenza (eds Capua, I. \& Mutinelli, F.). chap. Papi Editore, Bologna, pp. 13-20.

Caraco, T. \& Wang, I.N. (2008). Free-living pathogens: life-history constraints and strain competition. JTB, 250, 569-579.

Caraco, T., Glavanakov, S., Li, S., Maniatty, W. \& Szymanski, B.K. (2006). Spatially structured superinfection and the evolution of disease virulence. Theor. Popul. Biol., 69, 367-384.

Chen, H., Smith, G.J.D., Zhang, S.Y., Qin, K., Wang, J., Li, K.S. et al. (2005). Avian flu: H5N1 virus outbreak in migratory waterfowl. Nature, 436, 191-192.

Codeço, C. (2001). Endemic and epidemic dynamics of cholera: the role of the aquatic reservoir. BMC Infect. Dis., 1, 1-17.

Corelli, M. (1923). The Curse of Osiris. Daily News, London, 5th April, 1.

Costa, T.P., Brown, J.D., Howerth, E.W. \& Stallknecht, D.E. (2010). Effect of a prior exposure to a low pathogenic avian influenza virus in the outcome of a heterosubtypic low pathogenic avian influenza infection in mallards (Anas platyrbynchos). Avian Dis., 54, 1286-1291.

Cvjetanovic, B. \& Barua, D. (1972). The seventh pandemic of cholera. Nature, 239, 137-138.

Day, T. (2002). Virulence evolution via host exploitation and toxin production in spore-producing pathogens. Ecol. Lett., 5, 471-476.

Dieckmann, U., Metz, J., Sabelis, M. \& Sigmund, K. (eds) (2002). Adaptative dynamics of infectious diseases: In pursuit of virulence management. Cambridge University Press, Cambridge.

Dietz, K. (1976). The incidence of infectious diseases under the influence of seasonal fluctuations. Lect. Notes Biomath., 11, 1-15.

Errington, P.L. (1934). Vulnerability of bobwhite populations to predation. Ecology, 15, 110-127.

Eveillard, M., Martin, Y., Hidri, N., Boussougant, Y. \& Joly-Guillou, M.L. (2004). Carriage of methicillin-resistant staphylococcus aureus among hospital employees: prevalence, duration, and transmission to households. Infect. Control Hosp. Epidemiol., 25, 114-120.

Ewald, P.W. (1987). Pathogen-induced cycling of outbreaks in insect populations. Insect outbreaks (eds Barbosa, P.A. \& Schultz, J.C.). chap. Academic Press, London. pp. 269-286.

Ewald, P.W. (1991). Transmission modes and the evolution of virulence with special reference to cholera, influenza, and aids. Hum. Nat., 2, 1-30.

Ewald, P. (1993). Evolution of virulence. Scient. Am., 268, 86-93.

Ewald, P.W. (1994). The Evolution of Infectious Disease. Oxford University Press, Oxford.

Fenner, F. \& Ratcliffe, F.N. (1965). Myxomatosis. Cambridge University Press, Cambridge.

Gandon, S. (1998). The curse of the pharaoh hypothesis. Proc. Biol. Sci., 265, 15451552.

Keeling, M.J. \& Rohani, P. (2008). Modeling Infectious Diseases in Humans and Animals. Princeton University Press, Princeton.

King, A.A., Ionides, E.L., Pascual, M. \& Bouma, M.J. (2008). Inapparent infections and cholera dynamics. Nature, 454, 877-880.

Koelle, K., Cobey, S., Grenfell, B.T. \& Pascual, M. (2006). Epochal evolution shapes the phylodynamics of interpandemic influenza A (H3N2) in humans. Science, 314, 1898-1903.

Kooper, J.B., Morris, J.G. \& Levine, M.M. (1995). Cholera. Clin. Microbiol. Rev., Jan, 48-86.

Lipsitch, M., Colijn, C., Cohen, T., Hanage, W.P. \& Fraser, C. (2009). No coexistence for free: neutral null models for multistrain pathogens. Epidemics, 1, 2-13. 
Liu, J., Xiao, H., Lei, F., Zhu, Q., Qin, K., Zhang, X.W. et al. (2005). Highly pathogenic H5N1 influenza virus infection in migratory birds. Science, 309, 1206.

de Magny, G.C., Murtugudde, R., Sapiano, M.R.P., Nizam, A., Brown, C.W., Busalacchi, A.J. et al. (2008). Environmental signatures associated with cholera epidemics. Proc. Natl. Acad. Sci. U.S.A., 105, 17676-17681.

Maynard-Smith, J. (1982). Evolution and the Theory of Games. Cambridge University Press, Cambridge.

Nowak, M.A. (2006). Evolutionary Dynamics: Exploring the Equations of Life. Harvard University Press, Cambridge, MA.

Nowak, M.A. \& May, R.M. (1994). Superinfection and the evolution of parasite virulence. Proc. Biol. Sci., 255, 81-89. 133.

Olsen, B., Munster, V.J., Wallensten, A., Waldenström, J., Osterhaus, A.D.M.E. \& Fouchier, R.A.M. (2006). Global patterns of influenza a virus in wild birds. Science, 312, 384-388.

De Paepe, M. \& Taddei, F. (2006). Viruses' life history: towards a mechanistic basis of a trade-off between survival and reproduction among phages. PLoS Biol., 4, e193.

Park, A.W., Daly, J.M., Lewis, N.S., Smith, D.J., Wood, J.L.N. \& Grenfell, B.T. (2009). Quantifying the impact of immune escape on transmission dynamics of influenza. Science, 326, 726-728.

Parker, G. \& Smith, J.M. (1990). Optimality theory in evolutionary biology. Nature, $348,27-33$.

Pascual, M., Bouma, M.J. \& Dobson, A.P. (2002). Cholera and climate: revisiting the quantitative evidence. Microbes Infect., 4, 237-245.

Perdue, M.L., García, M., Senne, D. \& Fraire, M. (1997). Virulence-associated sequence duplication at the hemagglutinin cleavage site of avian influenza viruses. Virus Res., 49, 173-186.

Roche, B. \& Rohani, P. (2010). Environmental transmission scrambles coexistence patterns for avian influenza. Epidemics, 2, 92-98.

Roche, B., Lebarbenchon, C., Gauthier-Clerc, M., Chang, C.M., Thomas, F., Renaud, F. et al. (2009). Water-borne transmission drives avian influenza dynamics in wildbirds: the case of the 2005-2006 epidemics in the camargue area. Infect. Genet. Evol., 9, 800-805.
Rohani, P., Breban, R., Stallknecht, D.E. \& Drake, J.M. (2009). Environmental transmission of avian influenza viruses and its implications for disease control. Proc. Natl. Acad. Sci. U.S.A., 106(25), 10365-10369.

Walther, B.A. \& Ewald, P.W. (2004). Pathogen survival in the external environment and the evolution of virulence. Biol. Rev. Camb. Philos. Soc., 79, 849-869.

Webster, R.G., Bean, W.J., Gorman, O.T., Chambers, T.M. \& Kawaoka, Y. (1992). Evolution and ecology of influenza A viruses. Microbiol. Rev., 56, 152-179.

\section{SUPPORTING INFORMATION}

Additional Supporting Information may be found in the online version of this article:

Appendix S1 Evolutionary dynamics through genetic drift.

Appendix S2 Analysis without virulence.

Appendix S3 Influence of host demography.

Appendix S4 Influence of the mutation process.

Appendix S5 Influence of the ecological neutrality.

As a service to our authors and readers, this journal provides supporting information supplied by the authors. Such materials are peer-reviewed and may be re-organized for online delivery, but are not copy-edited or typeset. Technical support issues arising from supporting information (other than missing files) should be addressed to the authors.

Editor, John Jaenike

Manuscript received 5 January 2011

First decision made 2 February 2011

Manuscript accepted 20 March 2011 